\title{
Overexpressed FOXC2 in ovarian cancer enhances the epithelial-to-mesenchymal transition and invasion of ovarian cancer cells
}

\author{
BO LIU ${ }^{1}$, SHU-MEI HAN ${ }^{1}$, XIAO-YONG TANG ${ }^{1}$, LI HAN $^{1}$ and CHANG-ZHONG LI ${ }^{2}$ \\ ${ }^{1}$ Shandong Tumor Hospital and Institute, Jinan, Shandong $250117 ;{ }^{2}$ Shandong Provincial Hospital \\ Affiliated to Shandong University, Jinan, Shandong 250021, P.R. China
}

Received January 2, 2014; Accepted February 27, 2014

DOI: 10.3892/or.2014.3119

\begin{abstract}
Ovarian cancer is a highly invasive and metastatic disease with poor prognosis, particularly if this disease is diagnosed at an advanced stage, which is often the case. Researchers have argued that ovarian cancer cells that have undergone epithelial-to-mesenchymal transition (EMT) acquire aggressive malignant properties; however, the relevant molecular mechanisms in this setting are poorly understood. In cancer cases, the transcription factor forkhead box protein $\mathrm{C} 2$ (FOXC2) has been detected, but the function of this factor in ovarian cancer tumorigenesis remains unclear. In the present study, FOXC2 was overexpressed in invasive ovarian cancer cell lines and tissues. The invasive potential of ovarian cancer cells was significantly increased by ectopic FOXC2 expression but it was significantly decreased by RNA interference targeting FOXC2. E-cadherin and vimentin expression levels were modulated by FOXC2. These results indicated that FOXC2 was required for the maintenance of the mesenchymal phenotype after TGF- $\beta 1$ induced EMT in human ovarian cancer cells. Thus, FOXC2 or its associated gene expression program may provide an effective target for anti-EMT-based therapies. These therapies can then be performed to treat invasive ovarian tumor.
\end{abstract}

\section{Introduction}

Ovarian cancer, one of the leading causes of mortality involving gynecologic malignancies, is a highly metastatic disease characterized by widespread peritoneal dissemination and ascites (1). The development of new treatment protocols depends on the improved knowledge of the molecular mecha-

Correspondence to: Dr Chang-Zhong Li, Shandong Provincial Hospital Affiliated to Shandong University, Jinan, Shandong 250021, P.R. China

E-mail: slyylcz@126.com

Key words: forkhead box protein C2, ovarian cancer, epithelial-tomesenchymal transition, invasion nisms controlling metastasis as the treatment of patients in advanced stages yields low survival rates (2). Studies have shown that a morphological conversion known as epithelial-to-mesenchymal transition (EMT) is associated with the acquisition of malignant characteristics in ovarian cancer cells (3-9).

EMT was initially characterized as an important program during normal embryonic development $(10,11)$; however, studies have further suggested that carcinoma cells can reactivate the EMT program during tumor progression (12). Similar to cells that undergo EMT during normal development, carcinoma cells that undergo EMT lose cell-to-cell contacts, undergo major changes in their cytoskeleton, and acquire a mesenchymal-like morphology that enhances invasive and migratory abilities (13). Several signaling molecules present in tumor microenvironments can initiate EMT and metastasis; many of these same factors are aberrantly activated and/or overexpressed in human cancer (14-16).

Studies have revealed that EMT is governed by various regulatory networks. EMT is triggered by extracellular stimuli, such as TGF- $\beta 1$, fibroblast growth factor, hepatocyte growth factor and endothelin-1 $(6,7,17)$. These factors activate specific signaling pathways, thereby inducing changes in cytoskeletal organization and disrupting cell-to-cell junctions. In addition to these signaling pathways, transcription factors, such as Snail, Slug, Twist, Zeb1 and SIP1, are important in the promotion of EMT (18). Another example is forkhead box protein $\mathrm{C} 2$ (FOXC2), an EMT-inducing factor that has been detected in breast cancer (19). FOXC2 potently acts on mesenchymal tissues, suggesting that FOXC2 enables cancer cells to thrive in environments favorable for invasion and metastasis (20). Therefore, FOXC2 may act as an oncogene. However, whether or not FOXC2 is involved in ovarian cancer development and metastasis remains unknown.

This study showed that FOXC2 expression was increased in ovarian cancer tissues and cell lines. In human ovarian cancer cells, migration and invasion were significantly enhanced when FOXC2 was overexpressed. By contrast, these properties were inhibited when FOXC2 was knocked down. These results indicated that $\mathrm{FOXC} 2$ is required for the maintenance of a mesenchymal phenotype after TGF- $\beta 1$ induced EMT in human ovarian cancer cells. 


\section{Materials and methods}

Samples, cells and antibodies. Human normal ovarian and ovarian cancer tissue samples were provided by Shandong Provincial Hospital affiliated to Shandong University of China. All experiments were approved by the Ethics Committee of the Shandong Provincial Hospital affiliated to Shandong University and informed consent was obtained from all patients prior to specimen collection. ES-2 and SKOV3 cell lines were obtained from American Type Culture Collection (Manassas, VA, USA). NOS4, K2 and TAOV were established in the Department of Obstetrics and Gynecology, Nagoya University Graduate School of Medicine (21). OVSAHO was obtained from JCRB Cell Bank (Japanese Collection of Research Bioresources Cell Bank). All ovarian cancer cells were cultured in RPMI supplemented with $10 \%$ fetal bovine serum (FBS) and antibiotics. Mouse monoclonal FOXC2, E-cadherin and vimentin antibodies were purchased from Abcam (Cambridge, MA, USA). Mouse monoclonal $\beta$-actin antibody was purchased from Santa Cruz Biotechnology (Santa Cruz, CA, USA).

FOXC2-specific siRNA inhibition. To knock down FOXC2 expression in ovarian cancer cells, the following two siRNAs against FOXC2 were purchased from GenePharma (Shanghai, China): siFOXC2-no.1 (5'-GCAGTCTTATC TAACTATGATGCAA-3'), and siFOXC2-no.2 (5'-TCGCA AAGGGCATGAACTA-3'). Cells were grown in dishes until they reached $75 \%$ confluence and were then transfected for $24 \mathrm{~h}$ with siRNA specific to FOXC2 using the Lipofectamine 2000 transfection reagent, according to the manufacturer's instructions. FOXC2 expression was then confirmed by RT-PCR and western blot analyses.

Plasmid construct and generation of stable cell lines. Human cDNA of FOXC2 was cloned as previously reported (22). Fulllength cDNAs were subcloned into multiple cloning sites of the $\mathrm{pBabe}$ plasmid, forming the $\mathrm{pBabeFOXC2}$ expression plasmids. TAOV cells were transfected with the pBabe or pBabe-FOXC2 plasmid respectively, using the Lipofectamine 2000 according to the manufacturer's instructions (Invitrogen, Grand Island, NY, USA). Stable transfectants were obtained after selection with puromycin (10 $\mu \mathrm{g} / \mathrm{ml}$; Invitrogen) for 2 weeks. RT-PCR and western blot analyses were carried out to determine the expression of FOXC2 mRNA and protein in stable cell lines, respectively.

$R T-P C R$. Total RNA was extracted from tissues and cell lines using the TRIzol reagent (Invitrogen). Reverse transcription was performed using the Thermo script RT System (Invitrogen). Hot start PCR conditions were: $45 \mathrm{sec}$ at $94^{\circ} \mathrm{C}, 30 \mathrm{sec}$ at $55^{\circ} \mathrm{C}$, and $1 \mathrm{~min}$ at $72^{\circ} \mathrm{C}$ for 28 to 30 cycles or 26 cycles. This study used the following primers: FOXC2 (sense, 5'-CCTACC TGAGCGAGCAGAAT-3' and antisense, 5'-ACCTTGACGA AGCACTCGTT-3'); E-cadherin (sense, 5'-GGCACTTTTGAA GATCATTTTCTC-3' and antisense, 5'-CTGTGTTGAGGGC AATGAG-3'); vimentin (sense, 5'-GGCACTTTTGAAGATC ATTTTCTC-3' and antisense, 5'-CTGTGTTGAGGGCAAT GAG-3'); and GAPDH (sense, 5'-TGCCTCCTGCACCACCA ACT-3' and antisense, 5'-CCCGTTCAGCTCAGGGATGA-3').
Western blotting. Samples and cells were solubilized in radioimmunoprecipitation assay lysis buffer $[50 \mathrm{mmol} / 1$ Tris- $\mathrm{HCl}$ (pH 7.4), 1\% NP40, 0.25\% Na-deoxycholate, $150 \mathrm{mmol} / 1 \mathrm{NaCl}, 1 \mathrm{mmol} / \mathrm{l}$ EDTA, $1 \mathrm{mmol} / 1$ phenylmethylsulfonyl fluoride, $1 \mathrm{mg} / \mathrm{ml}$ each of aprotinin, leupeptin and pepstatin, $\left.1 \mathrm{mmol} / 1 \mathrm{Na}_{3} \mathrm{VO}_{4}, 1 \mathrm{mmol} / 1 \mathrm{NaF}\right]$. The supernatants, which contained the whole-cell protein extracts, were obtained after centrifugation of the cell lysates at $10,000 \mathrm{x} \mathrm{g}$ for $10 \mathrm{~min}$ at $4^{\circ} \mathrm{C}$. A total of $20 \mu \mathrm{g}$ of protein samples were loaded on a sodium dodecyl sulfate-PAGE gel (5\% stacking gel and $12 \%$ separating gel). These proteins were then transferred to polyvinylidene difluoride membranes (Millipore, Bedford, MA, USA). The membranes were initially probed with a primary antibody and then with a secondary antibody. The bound antibody was detected by enhanced chemiluminescence detection reagents (Amersham Biosciences, Piscataway, NJ, USA) according to the manufacturer's instructions. The band intensity was quantitated using ImageQuant software (Molecular Dynamics, Sunnyvale, CA, USA).

Confocal immunofluorescence microscopy. Cell lines were plated on culture slides (Costar, Manassas, VA, USA). After $24 \mathrm{~h}$, the cells were rinsed with PBS and fixed with $4 \%$ paraformaldehyde, and cell membrane was permeabilized using $0.5 \%$ Triton X-100. These cells were then blocked for $30 \mathrm{~min}$ in $10 \% \mathrm{BSA}$ and then incubated with primary antibodies overnight at $4^{\circ} \mathrm{C}$. After three washes in PBS, the slides were incubated for $1 \mathrm{~h}$ in the dark with FITC-conjugated secondary antibodies (Invitrogen). After three further washes, the slides were stained with DAPI for $5 \mathrm{~min}$ to visualize the nuclei, and examined using a Carl Zeiss confocal imaging system (LSM 780; Carl Zeiss, Jena, Germany).

Wound healing assay. Equal numbers of different cells were seeded in six-well tissue culture plates. When $90 \%$ confluence was reached, a single wound was created by gently removing the attached cells using a sterile plastic pipette tip. Debris was removed by washing the cells with serum-free medium. Cell migration of the cells into the wounded area was observed and noted at different time points. Various migrations and extended protrusion of cells from the border of the wound were visualized and photo-documented using an inverted microscope.

Cell invasion and motility assay. Cell invasion was measured in Matrigel-coated (BD, Franklin Lakes, NJ, USA) Transwell inserts $(6.5 \mathrm{~mm}$; Costar, Manassas, VA, USA) containing polycarbonate filters with $8 \mu \mathrm{m}$ pores as previously described in detail (23). The inserts were coated with $50 \mu 1$ of $1 \mathrm{mg} / \mathrm{ml}$ Matrigel matrix according to the manufacturer's recommendations. Cells $\left(2 \times 10^{5}\right)$ in $200 \mu \mathrm{l}$ of serum-free medium were plated in the upper chamber, whereas $600 \mu \mathrm{l}$ of medium with $10 \%$ FBS were added to the lower well. After $24 \mathrm{~h}$ incubation, the top cells were removed and the bottom cells were counted. The cells that migrated to the lower surface of the membrane were fixed in $4 \%$ paraformaldehyde and stained with $0.5 \%$ crystal violet. For each membrane, five random fields were counted at x10 magnification. The mean was calculated, and data are presented as means \pm SD from three independent experiments performed in triplicate. 
A
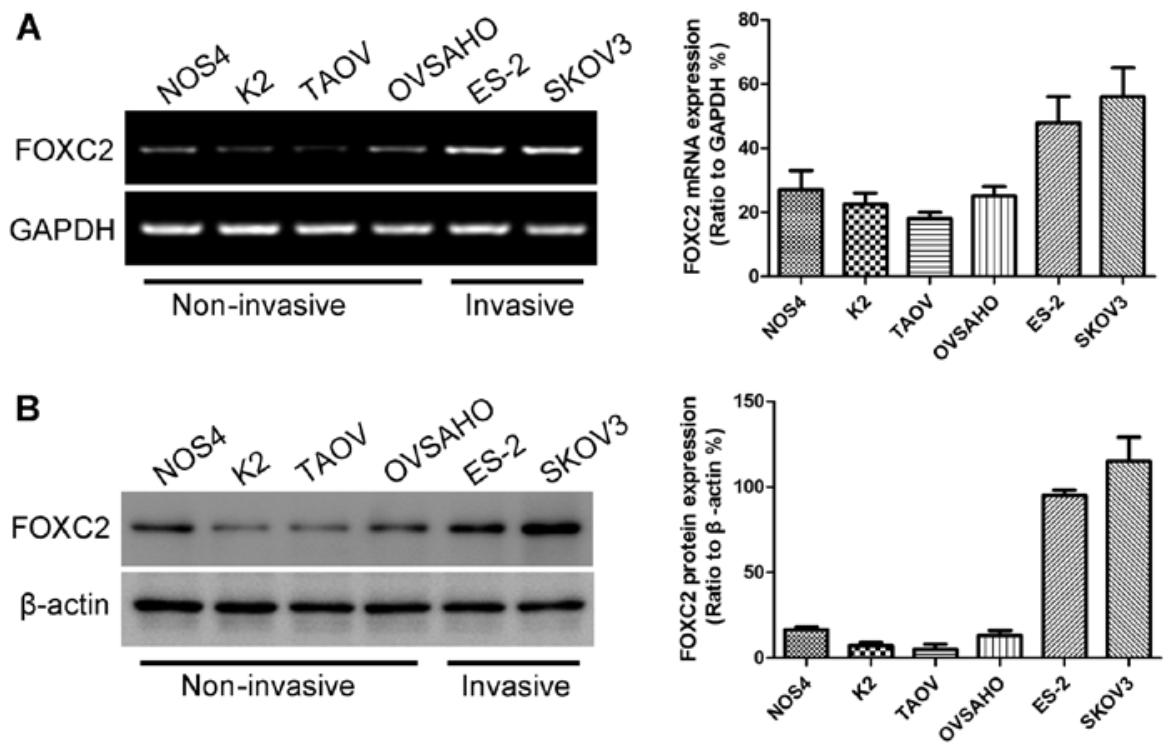

Figure 1. FOXC2 expression in non-invasive and invasive ovarian cancer cell lines. (A) Relative mRNA expression of FOXC2 in ovarian cancer cell lines examined by RT-PCR. GAPDH was used as an internal control. (B) Protein expression of FOXC2 in ovarian cancer cell lines examined by western blotting. $\beta$-actin was used as an internal control. The results were obtained from three independent experiments. Error bars indicate standard deviation.

Motility assays were performed using Transwell membrane inserts containing polycarbonate filters with $8 \mu \mathrm{m}$ pores. The methods used in the cell migration assay were similar to those in the Matrigel invasion assay, except that the Transwell insert was not coated with Matrigel.

Immunohistochemistry. Surgically excised specimens were fixed with $10 \%$ neutral formalin, embedded in paraffin, and $4 \mu \mathrm{m}$ specimen sections were prepared. Immunostaining was performed using the avidin-biotin-peroxidase complex method (UltrasensitiveTM; MaiXin Bio Co., Fuzhou, China). The sections were deparaffinized in xylene, rehydrated with graded alcohol, and boiled in $0.01 \mathrm{M}$ citrate buffer $(\mathrm{pH} 6.0)$ for $2 \mathrm{~min}$ with an autoclave. Hydrogen peroxide $(0.3 \%)$ was applied to block endogenous peroxide activity, and the sections were incubated with normal goat serum to reduce non-specific binding. Tissue sections were incubated with different monoclonal antibodies. Mouse immunoglobulin (with the same concentration of the antigen-specific antibody) was used as a negative control. Staining for both antibodies was performed at room temperature for $2 \mathrm{~h}$. Biotinylated goat anti-mouse serum $\mathrm{IgG}$ was used as a secondary antibody. The sections were washed and incubated with streptavidin-biotin conjugated with horseradish peroxidase. The peroxidase reaction was developed with 3,30-diaminobenzidine tetrahydrochloride. Two independent blinded investigators randomly examined all tumor slides. Five views were examined per slide, and 100 cells were observed per view at x400 magnification.

Statistical analysis. Experimental data are shown as the means \pm SD. A two-tailed Student's t-test was used to compare the results from the different treatment groups. Differences with $\mathrm{P}<0.05$ were considered statistically significant; SPSS/ Win11.0 software (SPSS, Inc., Chicago, IL, USA) was used to analyze the data.

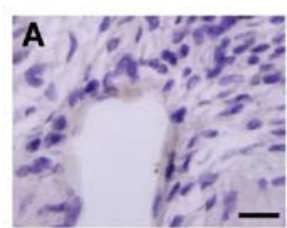

Normal

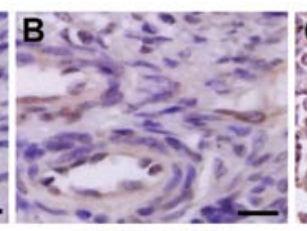

Non-invasive

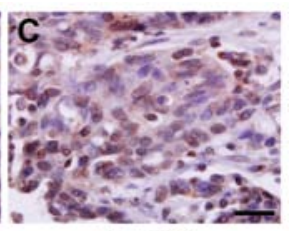

Invasive

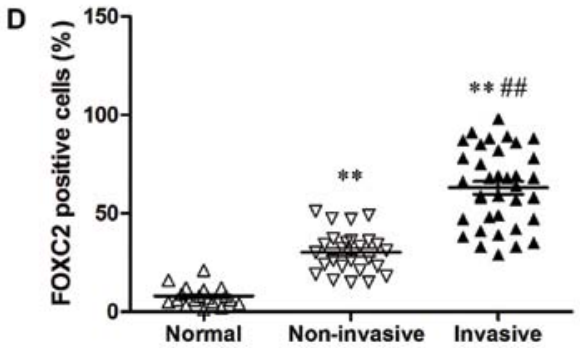

Figure 2. FOXC2 expression in paraffin-embedded sections from normal ovarian tissues and ovarian cancer tissues. (A) Immunohistochemistry analysis showing FOXC2 expression in normal ovarian tissues. (B) Immunohistochemistry analysis showing FOXC2 expression in non-invasive ovarian cancer tissues. (C) Immunohistochemistry analysis showing FOXC2 expression in invasive ovarian cancer tissues. (B and C) FOXC2 expression was primarily localized in the nuclei of tumor cells (in brown color). Stained FOXC2 was not observed in normal ovarian tissues. Scale bars, $50 \mu \mathrm{m}$. (D) Ratio of FOXC2 positive cells in normal ovarian tissues, non-invasive ovarian cancer tissues and invasive ovarian cancer tissues. ${ }^{* *} \mathrm{P}<0.01 \mathrm{vs}$. normal ovarian tissues and ${ }^{\# \#} \mathrm{P}<0.001$ vs. non-invasive ovarian cancer tissues are based on Student's t-test. The results were obtained from three independent experiments. Error bars indicate standard deviation.

\section{Results}

FOXC2 is overexpressed in metastatic ovarian cancer cell lines and invasive ovarian cancer tissues. To identify whether or not FOXC2 can initiate ovarian cancer invasion and metastasis, we initially evaluated the mRNA expression levels of 
A

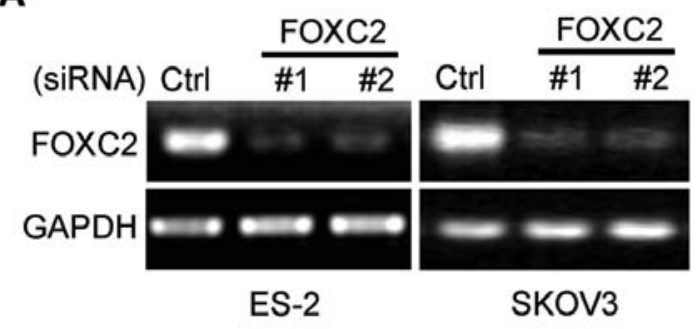

C

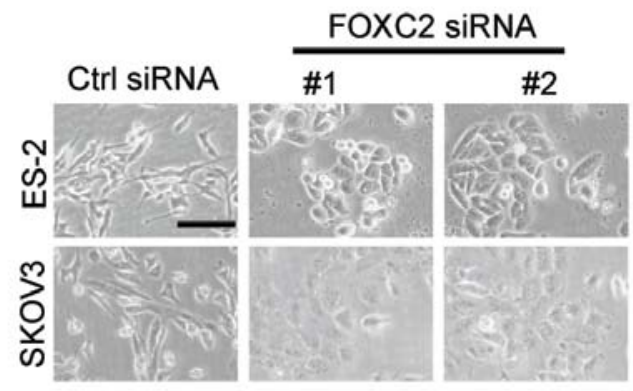

E

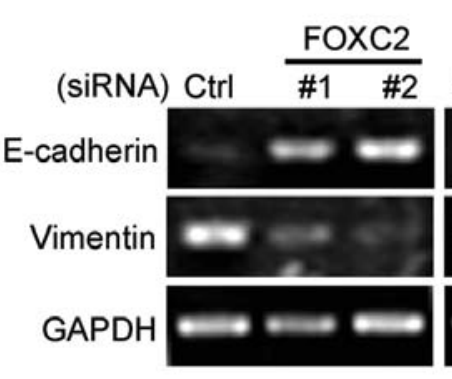

ES-2

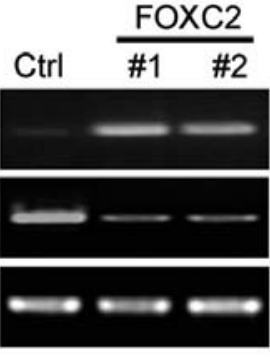

SKOV3
B

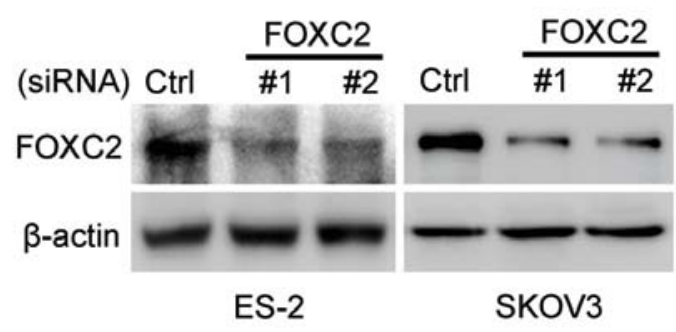

D

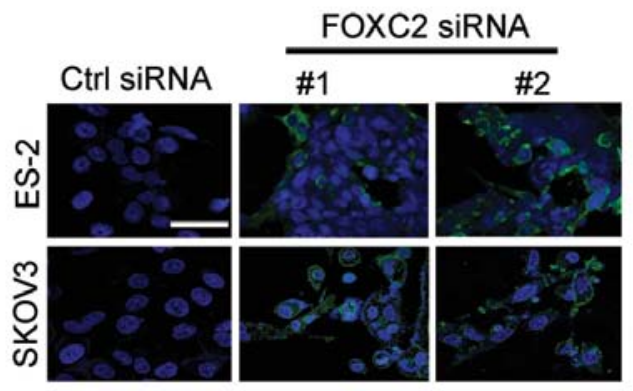

$\mathbf{F}$

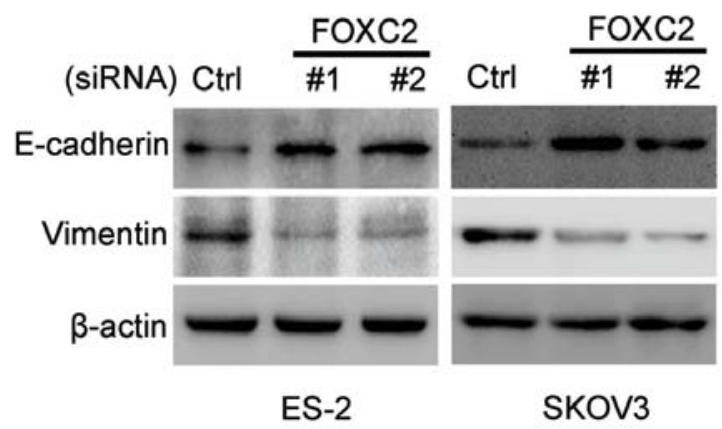

Figure 3. Depletion of FOXC2 induces the reversion of EMT. After siRNA transfection was performed, FOXC2 expression was examined by (A) RT-PCR and (B) western blotting in ES-2 and SKOV3 cell lines. (C) Cells were transfected with control cells or FOXC2 siRNA; images were obtained after 72 h to visualize cellular morphology. Scale bar, $50 \mu \mathrm{m}$. (D) Cells were cultured on glass cover slips and transfected with siRNA. After $72 \mathrm{~h}$, cells were fixed and immunostained with an anti-E-cadherin antibody and DAPI (green, E-cadherin; blue, DAPI). Scale bar, $50 \mu \mathrm{m}$. (E) Total RNA was extracted from siRNA-transfected cells, and the mRNA expression levels of the indicated genes were determined by RT-PCR. (F) After siRNA transfection was performed, the expression of the indicated proteins was examined by western blotting. The results were obtained from three independent experiments.

FOXC2 in six ovarian cancer cell lines (NOS4, K2, TAOV, OVSAHO, ES-2 and SKOV3) by conducting RT-PCR analysis. The highest FOXC2 expression was observed in ES-2 and SKOV 3 cells (Fig. 1A). The mRNA expression level of FOXC2 was related to the malignant characteristics of ovarian cancer cells. ES-2 and SKOV3 cells were more invasive than other ovarian cancer cell lines and could grow under anchorageindependent conditions (24). Western blotting results also showed the protein expression levels of FOXC2 in the six ovarian cancer cell lines (Fig. 1B).

FOXC2 expression was also analyzed in normal ovarian tissues and cancer tissues by immunohistochemistry (normal ovarian tissues, 18; non-invasive ovarian cancer tissues, 26; invasive ovarian cancer tissues, 36). Human normal ovarian tissues did not show immunostaining properties (Fig. 2A). FOXC2 protein, which was localized in the nuclei of tumor cells, was expressed in all the human ovarian cancer samples (Fig. 2B and C). We also found a strong expression of FOXC2 protein in some cancer tissues, and this expression was correlated with metastatic property (Fig. 2D). These associations suggested that FOXC2 expression was induced during tumor progression. FOXC2 may also exhibit a causal function, enabling metastatic dissemination.

FOXC2 depletion induces the reversion of EMT. We determined whether or not the expression of FOXC2 contributes to the EMT of ovarian cancer cells. We suppressed FOXC2 expression in the cells with high FOXC2 expression (ES-2 and SKOV3) by constructing two siRNA oligonucleotides targeting the mRNA of human FOXC2. The two siRNAs of FOXC2 reduced the level of FOXC2 expression at mRNA and protein levels by $>70 \%$ (Fig. 3A and B).

We then examined the effects of FOXC2 depletion in the cell lines with high FOXC2 expression. FOXC2 depletion induced the recovery of cell-to-cell adhesions; the cellular morphologies of these cell lines were similar to those of epithelial cells (Fig. 3C). The results of immunostaining analysis revealed that a clear recovery of E-cadherin localization was 
A

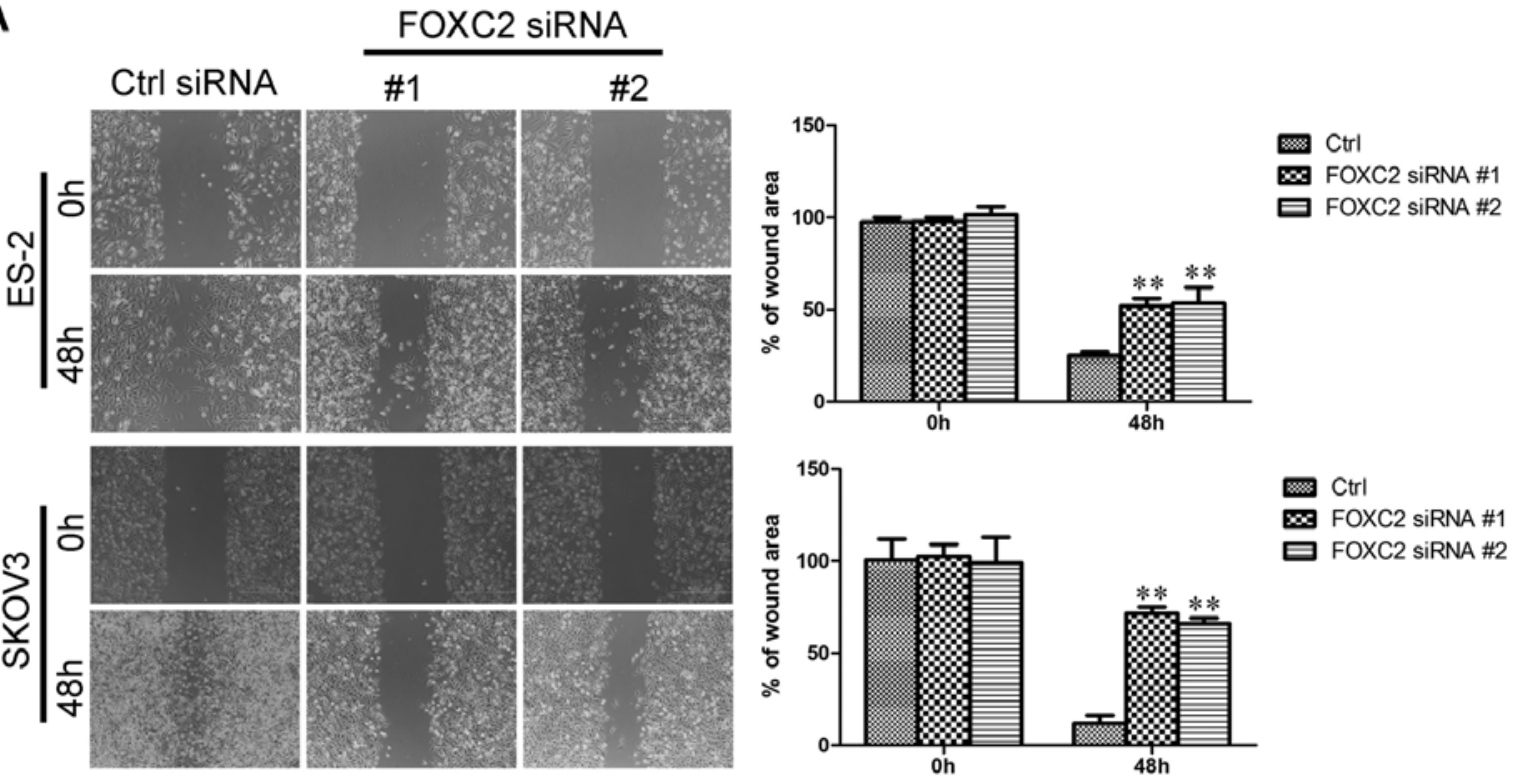

B
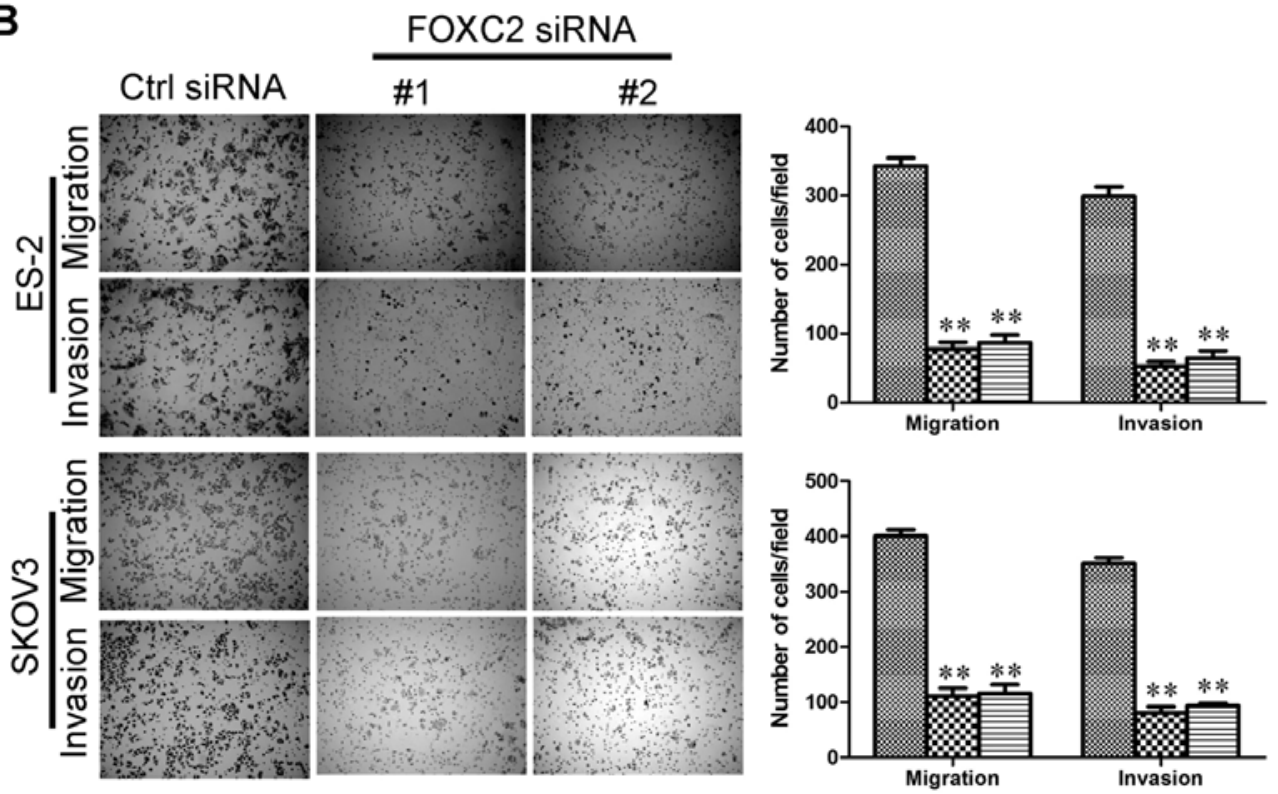

Figure 4. Depleted FOXC2 inhibits ovarian cancer cell migration and invasion. (A) ES-2 and SKOV3 cells were transfected with siRNA and then subjected to wound healing assay after $48 \mathrm{~h}$. Uncovered areas in the wound healing assays were quantified as the percentage of the original wound area (A, right). (B) ES-2 and SKOV3 cells were transfected with siRNA and then subjected to Transwell migration (top) and Matrigel invasion (bottom) assays after $48 \mathrm{~h}$. Cells migrating through the membrane and cells invading the Matrigel in each group were quantified and are shown as proportions of the corresponding control cells. ${ }^{* *} \mathrm{P}<0.01$ is based on Student's t-test. The results were obtained from three independent experiments. Error bars indicate standard deviation.

induced in ES-2 and SKOV3 cells by knocking down FOXC2 expression (Fig. 3D). This result suggested that the cells underwent a reversion of EMT when FOXC2 level was reduced in ES-2 and SKOV3 cells. To test this hypothesis, we examined the expression levels of epithelial and mesenchymal markers. The upregulation of E-cadherin and the downregulation of vimentin were detected at mRNA and protein levels in ES-2 and SKOV3 cells (Fig. 3E and F). These results indicated that FOXC2 is important for SKOV3 and ES-2 cells to maintain mesenchymal characteristics.

FOXC2 depletion inhibits ovarian cancer cell migration and invasion. EMT is associated with malignant properties, such as migration and invasion $(25,26)$. We investigated whether or not FOXC2 is required for these properties in ovarian cancer cells. The effect of FOXC2 on cell migration was initially assessed by wound healing assay. ES-2 siFOXC2 and SKOV3 siFOXC2 cells exhibited a significantly slower closure of the wound area than the control cells (Fig. 4A). This result was confirmed by Boyden's chamber assay (Fig. 4B). Moreover, the invasion of SKOV3 and ES-2 cells through the Matrigel was significantly suppressed by FOXC2 knockdown (Fig. 4B).

Ectopic FOXC2 expression induces EMT and promotes the migration and invasion of ovarian cancer cells in vitro. To evaluate the oncogenic activity of FOXC2 in ovarian cancer, we 


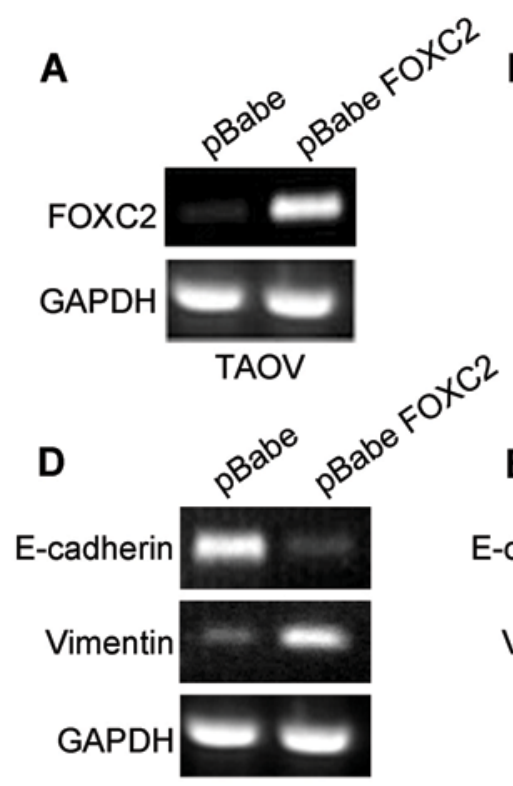

B
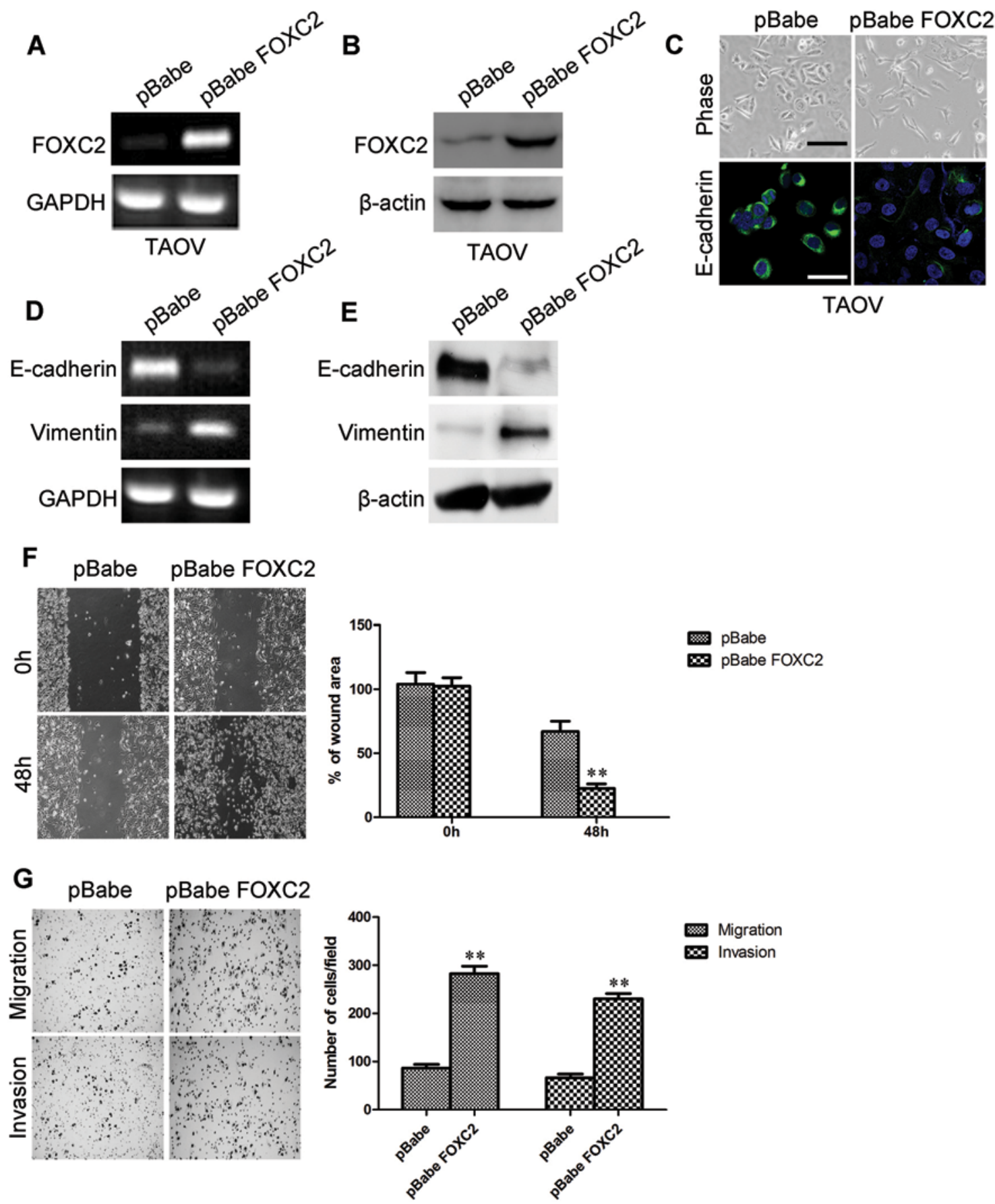

Figure 5. Ectopic FOXC2 expression induces EMT and promotes the migration and invasion of ovarian cancer cells in vitro. Retrovirally established stable overexpression of FOXC2 in TAOV cells (designated as TAOV-pBabe FOXC2). FOXC2 expression level in the resultant cell line was verified by (A) RT-PCR and (B) western blotting. Images of TAOV-pBabe and TAOV-pBabe FOXC2 cells were obtained to visualize cellular morphology (C, top; scale bar, $50 \mu \mathrm{m})$. Cells were cultured on glass cover slips and immunostained with an anti-E-cadherin antibody and DAPI (green, E-cadherin; blue, DAPI; C, bottom; scale bar, $30 \mu \mathrm{m}$ ). (D) Total RNA was extracted from TAOV-pBabe and TAOV-pBabe FOXC2 cells, and the mRNA expression levels of the indicated genes were determined by RT-PCR. (E) Expression levels of the indicated proteins were examined by western blotting. (F) TAOV-pBabe and TAOV-pBabe FOXC2 cells were subjected to wound healing assay. Uncovered areas in the wound healing assays were quantified as the percentage of the original wound area (right), (G) TAOV-pBabe and TAOV-pBabe FOXC2 cells were subjected to Transwell migration (top) and Matrigel invasion (bottom) assays. Cells migrating through the membrane and cells invading through the Matrigel in each group were quantified and are shown as proportions of their corresponding control cells. ${ }^{* *} \mathrm{P}<0.01$ is based on Student's t-test. The results were obtained from three independent experiments. Error bars indicate standard deviation.

retrovirally established the stable overexpression of FOXC2 in TAOV cells (designated as TAOV-pBabe FOXC2). The FOXC2 expression in the resultant cell line was verified by RT-PCR (Fig. 5A) and western blotting (Fig. 5B). We initially observed the morphological changes and found that TAOV-pBabe FOXC2 cells exhibited a fibroblastic morphology (Fig. 5C).
This observation was further confirmed by analyzing the expression levels of epithelial and mesenchymal markers. We showed that FOXC2 overexpression decreased the levels of epithelial markers (E-cadherin; Fig. 5C and E) and increased the levels of mesenchymal markers (vimentin; Fig. 5E) in TAOV-pBabe FOXC2 cells. The mRNA expression levels were 
A

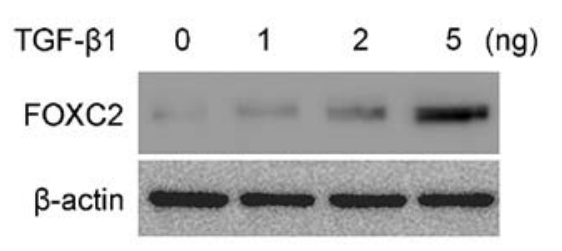

C

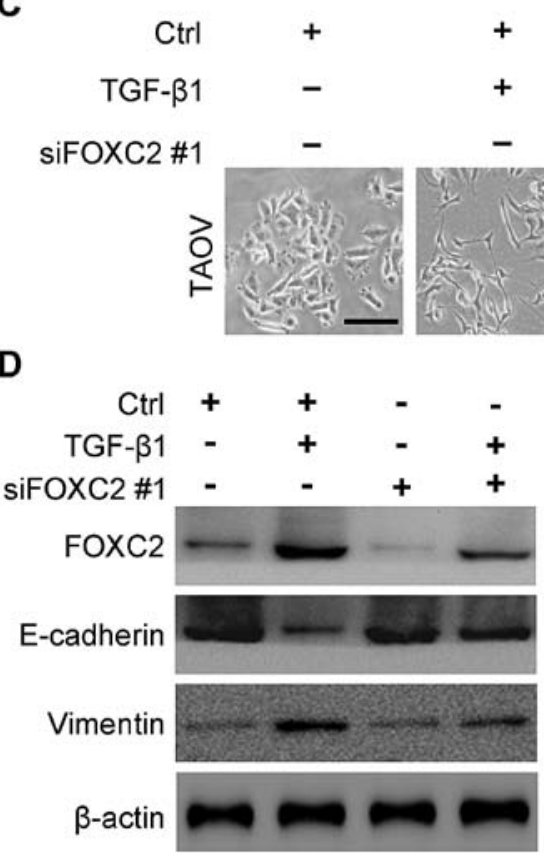

B
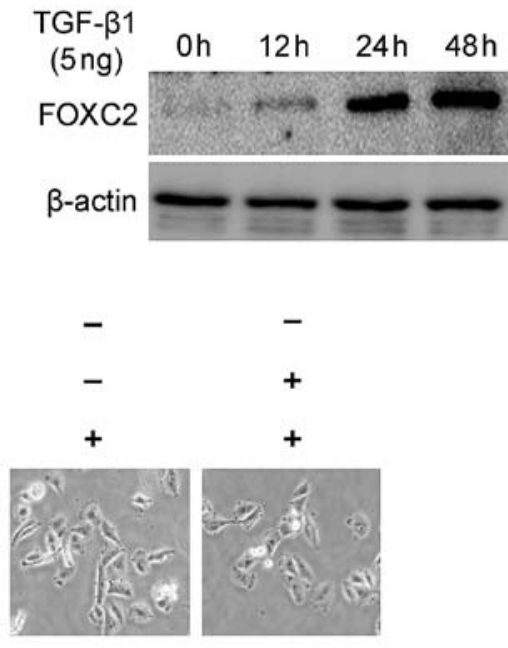

Figure 6. TGF- $\beta 1$ stimulation induces the upregulation of FOXC 2 and FOXC2 suppression attenuates TGF- $\beta 1$-induced EMT in TAOV cells. FOXC2 expression was measured by western blotting. (A) FOXC2 expression was upregulated by TGF- $\beta 1$ at different time-points in TAOV cells compared with untreated cells. (B) FOXC2 expression was upregulated by TGF- $\beta 1$ in a concentration-dependent manner in TAOV cells compared with the untreated cells. (C) TAOV cells were transfected with FOXC2 siRNA \#1 or were treated with TGF- $\beta 1$; images were obtained after $48 \mathrm{~h}$ to visualize cellular morphology. (D) After $48 \mathrm{~h}$ of treatment, the expression of the indicated proteins was examined by western blotting. The results were obtained from three independent experiments. Scale bar, $50 \mu \mathrm{m}$.

correlated with the corresponding protein levels (Fig. 5D), suggesting that FOXC2 affected the expression of epithelial and mesenchymal markers at the transcript level.

The effect of FOXC2 on cell migration was initially assessed by wound healing assay. TAOV-pBabe FOXC2 cells exhibited a significantly faster closure of the wound area than the control cells (Fig. 5F). This result was confirmed by Boyden's chamber assay; TAOV-pBabe FOXC2 cells migrated by more than two-fold in the Transwell membranes than the control cells after $48 \mathrm{~h}$ of incubation (Fig. 6F). TAOV-pBabe FOXC2 cells also exhibited a higher degree of invasion in the Matrigel (Fig. 6F). These results indicated that FOXC2 promoted the migratory and invasive behaviors of ovarian cancer cells.

TGF- $\beta 1$ stimulation induces FOXC2 upregulation. Although various growth factors and cytokines orchestrate EMT, TGF- $\beta 1$ exhibits a major function in this process $(27,28)$. Considering that FOXC2 is associated with EMT, we then investigated whether or not the expression of FOXC2 is upregulated by TGF- $\beta 1$ in ovarian cancer cells (TAOV). TGF- $\beta 1$ increased FOXC2 expression at $12 \mathrm{~h}$ after treatment and reached the highest level at $24 \mathrm{~h}$ (Fig. 6A) compared with the untreated cells. We also found that the increasing FOXC2 expression stimulated by TGF- $\beta 1$ was concentration-dependent. Hence, FOXC2 level increased gradually as TGF- $\beta 1$ level increased (Fig. 6B).

FOXC2 suppression attenuates TGF- $\beta 1$-induced EMT in TAOV cells. Considering that TGF- $\beta 1$ stimulation can induce EMT and upregulate FOXC2 expression in TAOV cells, we proposed that FOXC2 may be involved in TGF- $\beta 1$-induced EMT of TAOV cells. To understand the specific biological functions that FOXC2 performs during TGF- $\beta 1$-induced EMT, we knocked down FOXC2 in TAOV cells transfected with a FOXC2-targeting siRNA. We initially observed the morphological changes in TGF- $\beta 1$-induced TAOV cells after FOXC2 silencing was performed. siRNA-transfected cells showed epithelial morphology (Fig. 6C) compared with the controltransfected cells. FOXC2 expression was then observed by western blotting after $48 \mathrm{~h}$ of TGF- $\beta 1$ treatment. In Fig. 6D, FOXC2 expression in FOXC2 siRNA-transfected cells was downregulated significantly compared with that in the control-transfected cells. These results showed that FOXC2 levels were decreased in FOXC2 siRNA-transfected cells and the silencing of FOXC2 expression reversed TGF- $\beta 1$-induced morphological transition (Fig. 6C). We subsequently determined the underlying molecular mechanism. In Fig. 6D, FOXC2 expression was detected after TGF- $\beta 1$ was stimulated for $48 \mathrm{~h}$ in the cells transfected with FOXC2 siRNA or with the control cells. The FOXC2 expression in FOXC2 siRNA-transfected cells significantly decreased compared with that of the control cells. These results showed that siRNA against FOXC2 could efficiently reduce FOXC2 expression. At the same time, the downregulation of FOXC2 resulted in a 
A
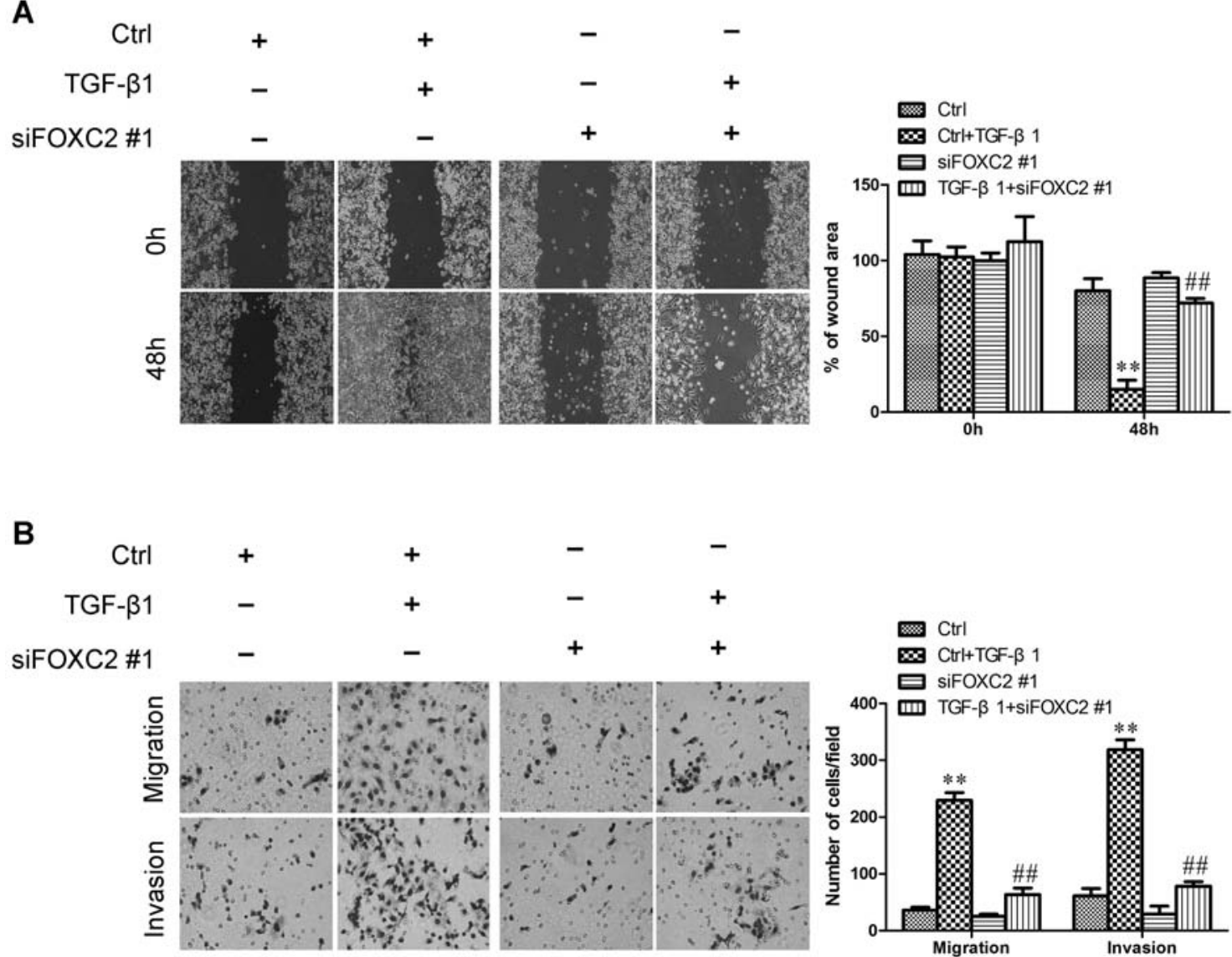

Figure 7. FOXC2 suppression effectively inhibits TGF- $\beta 1$-induced migration and invasion of TAOV cells. (A) TAOV cells were transfected with siRNA or were treated with TGF- $\beta 1$ and then subjected to wound healing assay after $48 \mathrm{~h}$. Uncovered areas in the wound healing assays were quantified as the percentage of the original wound area (A, right). (B) TAOV cells were transfected with siRNA or treated with TGF- $\beta 1$ and then subjected to Transwell migration (top) and Matrigel invasion (bottom) assays. Cells migrating through the membrane and cells invading the Matrigel in each group were quantified and are shown as proportions of the control cells. ${ }^{* *} \mathrm{P}<0.01$ is based on Student's $\mathrm{t}$-test. The results were obtained from three independent experiments. Error bars indicate standard deviation.

decrease in the mesenchymal marker vimentin and an increase in the epithelial marker E-cadherin (Fig. 6D).

FOXC2 suppression effectively suppresses the TGF- $\beta 1$ induced migration and invasion of TAOV cells. Considering that EMT can increase cell motility, we then examined whether or not FOXC2 can modulate the migratory and invasive abilities of TGF- $\beta 1$-induced ovarian cancer cells. To determine the functional changes in the behavior of TAOV cells after FOXC2 is suppressed, we initially assessed the effect of FOXC2 on TGF- $\beta 1$-induced cell migration by conducting a wound healing assay. The cells were treated with TGF- $\beta 1$; as a result, the cells were stimulated and migrated, thereby closing the wound. By comparison, TGF- $\beta 1$-mediated migration was reversed when the cells were co-incubated with siFOXC2. Mock treatment alone did not markedly affect cell migration (Fig. 7A). This result was further evaluated by Boyden's chamber assay, in which the cells migrated through the Transwell membranes to a lesser extent than their TGF- $\beta 1$-induced cells after $48 \mathrm{~h}$ of incubation (Fig. 7B). Moreover, FOXC2 suppression markedly reduced the invasion ability through the Matrigel (Fig. 7B). These results indicated that FOXC2 suppression effectively inhibited the TGF- $\beta 1$-induced migration of TAOV cells.

\section{Discussion}

To the best of our knowledge, the present study is the first to show that FOXC2 is overexpressed in ovarian cancer cells and is involved in ovarian cancer cell migration and invasion. FOXC2 overexpression in ovarian cancer cells induced EMT, migration, and invasion in vitro. By contrast, silencing FOXC2 aggressively reversed these events and invaded the ovarian cancer cells. We also showed that TGF- $\beta 1$ stimulation induced the upregulation of FOXC2. Furthermore, FOXC2 exhibited an important function in the TGF- $\beta 1$-induced EMT of ovarian cancer cells. On the basis of these results, we proposed a model for FOXC2 regulation of EMT and metastasis of ovarian cancer.

Ovarian cancer is a major cause of cancer-related mortality among women as this disease is typically not diagnosed until the disease is at advanced stages when cancer is highly metastatic (1). Although current methodologies (surgery, radiation and chemotherapy) are considered relatively effective for the treatment of primary ovarian tumors, many patients treated at advanced stages eventually suffer recurrence at metastatic sites (29). Considering that cancer metastasis is possibly initiated by EMT, researchers focused on the identification of possible therapeutic agents that may retard or reverse EMT (30). 
A high incidence of FOXC2 expression has been observed in some types of cancer, and such expression has been significantly associated with metastatic disease; however, limited information is available on the function of FOXC2 expression in ovarian cancer (20,31-33). Previous studies showed that FOXC2 is important for vascular formation during development (34). Other studies have shown that FOXC2 is expressed in the endothelium of tumors in humans and mice $(22,35)$. The present study is the first to define the functional roles of human FOXC2 in ovarian cancer.

Motility and invasion are major events in cancer metastasis; such events are also associated with poor prognosis in patients with cancer (36). However, the mechanisms associated with cell invasiveness remain poorly understood. Metastasis is a complex phenomenon regulated by many components that facilitate the detachment of tumor cells from primary tumors to secondary sites (36). EMT promotes the distribution of a single carcinoma cell from primary tumor sites to distant organs (metastasis); EMT also exhibits a major function in ovarian cancer progression (37). Another study showed that FOXC2 is a transcription factor that can induce EMT (19). Our study further demonstrated that FOXC2 promotes the migratory and invasive potential of ovarian cancer cells. The ectopic FOXC2 expression in ovarian cancer cells increased the migratory and invasive behaviors in vitro. This observation is consistent with that in a previous study, in which FOXC2 was involved in the EMT of breast cancer cells (19). The findings of our study revealed an important function of FOXC2 in the tumorigenesis and metastasis of ovarian cancer cells.

TGF- $\beta 1$ is a pleiotropic factor that exhibits a physiological function in regulating proliferation, differentiation, development, wound healing and angiogenesis (27). In addition, TGF- $\beta 1$-induced EMT has been well established as an important mechanism of ovarian cancer progression (38). However, TGF- $\beta 1$-induced EMT is a very complicated process, and the exact function of TGF- $\beta 1$ in the stimulation of EMT is poorly understood. In the current study, ovarian cancer cells underwent EMT changes after these cells were exposed to TGF- $\beta 1$. Our data also showed an increased ability of TGF- $\beta 1$-induced TAOV cells to undergo cell migration and invasion compared with that of the untreated cells. These results are consistent with those in previous studies. We also found that TGF- $\beta 1$-treated TAOV cells showed a high FOXC2 expression compared with the untreated cells. Our novel finding is noteworthy as it connects two very important molecules, namely, TGF- $\beta 1$ and TAOV, of the developmental pathway to ovarian tumor aggressiveness. Our result is also consistent with that in previously published reports, in which the function of EMT in tumor aggressiveness and metastasis was demonstrated.

Our results also suggested that the maintenance of EMT phenotype in TGF- $\beta 1$-treated cells may be related to the sustained activation of FOXC2. The inhibition of TGF- $\beta 1$-induced FOXC2 by siRNA decreased the ability of TGF- $\beta 1$-treated TAOV cells to migrate and invade.

We further showed the importance of FOXC2 in EMT, in which the siRNA-inhibited FOXC2 signaling could downregulate mesenchymal markers, such as vimentin. This result is consistent with the upregulation of epithelial markers, such as E-cadherin. These results also suggested that the attenua- tion of FOXC2 signaling could reverse the EMT phenotype to mesenchymal-to-epithelial transition, resulting in decreased cell migration, invasion and tumorigenic potential. Our data showed for the first time that TGF- $\beta 1$-induced EMT is mediated by upregulating FOXC2 as the knockdown of FOXC2 by FOXC2-specific siRNA significantly attenuated EMT induction by TGF- $\beta 1$ treatment.

In conclusion, this study is the first to show that FOXC2 promotes ovarian cancer cell proliferation and invasion properties. FOXC2 also exhibited an important function in the enhancement of malignancy by TGF- $\beta 1$ in ovarian cancer cells. The results of this study could be used as guidelines to target novel proteins located downstream of FOXC2 and disrupt the signaling pathways involved in the proliferation, motility and invasion of ovarian cancer.

\section{Acknowledgements}

This project was supported by the Natural Science Foundation of China (81172180).

\section{References}

1. Naora $\mathrm{H}$ and Montell DJ: Ovarian cancer metastasis: integrating insights from disparate model organisms. Nat Rev Cancer 5: 355-366, 2005.

2. Schmitt J and Matei D: Targeting angiogenesis in ovarian cancer. Cancer Treat Rev 38: 272-283, 2012.

3. Davidson B, Tropé CG and Reich R: Epithelial-mesenchymal transition in ovarian carcinoma. Front Oncol 2: 33, 2012.

4. Comamala M, Pinard M, Theriault C, et al: Downregulation of cell surface CA125/MUC16 induces epithelial-to-mesenchymal transition and restores EGFR signalling in NIH:OVCAR3 ovarian carcinoma cells. Br J Cancer 104: 989-999, 2011.

5. Cheng JC, Auersperg N and Leung PC: EGF-induced EMT and invasiveness in serous borderline ovarian tumor cells: a possible step in the transition to low-grade serous carcinoma cells? PLoS One 7: e34071, 2012.

6. Cheng JC, Auersperg N and Leung PC: TGF-beta induces serous borderline ovarian tumor cell invasion by activating EMT but triggers apoptosis in low-grade serous ovarian carcinoma cells. PLoS One 7: e42436, 2012.

7. Bagnato A and Rosanò L: Epithelial-mesenchymal transition in ovarian cancer progression: a crucial role for the endothelin axis. Cells Tissues Organs 185: 85-94, 2007.

8. Ahmed N, Thompson EW and Quinn MA: Epithelial-mesenchymal interconversions in normal ovarian surface epithelium and ovarian carcinomas: an exception to the norm. J Cell Physiol 213: 581-588, 2007.

9. Elloul S, Vaksman O, Stavnes HT, Tropé CG, Davidson B and Reich R: Mesenchymal-to-epithelial transition determinants as characteristics of ovarian carcinoma effusions. Clin Exp Metastasis 27: 161-172, 2010.

10. Yilmaz $\mathrm{M}$ and Christofori G: EMT, the cytoskeleton, and cancer cell invasion. Cancer Metastasis Rev 28: 15-33, 2009.

11. Onder TT, Gupta PB, Mani SA, Yang J, Lander ES and Weinberg RA: Loss of E-cadherin promotes metastasis via multiple downstream transcriptional pathways. Cancer Res 68: 3645-3654, 2008.

12. Wang Y,Wen M, Kwon Y, et al: CUL4A induces epithelial-mesenchymal transition and promotes cancer metastasis by regulating ZEB1 expression. Cancer Res 74: 520-532, 2013.

13. Huang RY, Chung VY and Thiery JP: Targeting pathways contributing to epithelial-mesenchymal transition (EMT) in epithelial ovarian cancer. Curr Drug Targets 13: 1649-1653, 2012.

14. Wang Y, Liu Y, Lu J, et al: Rapamycin inhibits FBXW7 lossinduced epithelial-mesenchymal transition and cancer stem cell-like characteristics in colorectal cancer cells. Biochem Biophys Res Commun 434: 352-356, 2013.

15. Lu J, Wen M, Huang Y, et al: C2ORF40 suppresses breast cancer cell proliferation and invasion through modulating expression of M phase cell cycle genes. Epigenetics 8: 571-583, 2013. 
16. Wang Y, Ma G, Wang Q, et al: Involvement of CUL4A in regulation of multidrug resistance to $\mathrm{P}$-gp substrate drugs in breast cancer cells. Molecules 19: 159-176, 2013.

17. Rosano L, Spinella F, Di Castro V, et al: Endothelin-1 promotes epithelial-to-mesenchymal transition in human ovarian cancer cells. Cancer Res 65: 11649-11657, 2005.

18. Peinado H, Olmeda D and Cano A: Snail, Zeb and bHLH factors in tumour progression: an alliance against the epithelial phenotype? Nat Rev Cancer 7: 415-428, 2007.

19. Hollier BG, Tinnirello AA, Werden SJ, et al: FOXC2 expression links epithelial-mesenchymal transition and stem cell properties in breast cancer. Cancer Res 73: 1981-1992, 2013.

20. Nishida N, Mimori K, Yokobori T, et al: FOXC2 is a novel prognostic factor in human esophageal squamous cell carcinoma. Ann Surg Oncol 18: 535-542, 2011.

21. Kajiyama H, Kikkawa F, Suzuki T, Shibata K, Ino K and Mizutani S: Prolonged survival and decreased invasive activity attributable to dipeptidyl peptidase IV overexpression in ovarian carcinoma. Cancer Res 62: 2753-2757, 2002.

22. Mortazavi F, An J, Dubinett S and Rettig M: p120-catenin is transcriptionally downregulated by FOXC2 in non-small cell lung cancer cells. Mol Cancer Res 8: 762-774, 2010.

23. Fu J, Lv X, Lin $\mathrm{H}$, et al: Ubiquitin ligase cullin 7 induces epithelial-mesenchymal transition in human choriocarcinoma cells. J Biol Chem 285: 10870-10879, 2010.

24. Yuan H, Kajiyama H, Ito S, et al: ALX1 induces snail expression to promote epithelial-to-mesenchymal transition and invasion of ovarian cancer cells. Cancer Res 73: 1581-1590, 2013.

25. Tiwari N, Gheldof A, Tatari M and Christofori G: EMT as the ultimate survival mechanism of cancer cells. Semin Cancer Biol 22: 194-207, 2012.

26. Schmalhofer O, Brabletz S and Brabletz T: E-cadherin, betacatenin, and ZEB1 in malignant progression of cancer. Cancer Metastasis Rev 28: 151-166, 2009.

27. Katsuno Y, Lamouille S and Derynck R: TGF-beta signaling and epithelial-mesenchymal transition in cancer progression. Curr Opin Oncol 25: 76-84, 2013.
28. Wendt MK, Allington TM and Schiemann WP: Mechanisms of the epithelial-mesenchymal transition by TGF-beta. Future Oncol 5: 1145-1168, 2009.

29. Nougaret S, Addley HC, Colombo PE, et al: Ovarian carcinomatosis: how the radiologist can help plan the surgical approach Radiographics 32: 1775-1803, 2012.

30. Tsai JH and Yang J: Epithelial-mesenchymal plasticity in carcinoma metastasis. Genes Dev 27: 2192-2206, 2013.

31. Zhu JL, Song YX, Wang ZN, et al: The clinical significance of mesenchyme forkhead 1 (FoxC2) in gastric carcinoma. Histopathology 62: 1038-1048, 2013.

32. Watanabe T, Kobunai T, Yamamoto Y, et al: Gene expression of mesenchyme forkhead 1 (FOXC2) significantly correlates with the degree of lymph node metastasis in colorectal cancer. Int Surg 96: 207-216, 2011.

33. Mani SA, Yang J, Brooks M, et al: Mesenchyme Forkhead 1 (FOXC2) plays a key role in metastasis and is associated with aggressive basal-like breast cancers. Proc Natl Acad Sci USA 104: 10069-10074, 2007.

34. Seo S, Fujita H, Nakano A, Kang M, Duarte A and Kume T: The forkhead transcription factors, Foxc1 and Foxc2, are required for arterial specification and lymphatic sprouting during vascular development. Dev Biol 294: 458-470, 2006.

35. Cederberg A, Grände M, Rhedin M, Peng XR and Enerbäck S In vitro differentiated adipocytes from a Foxc2 reporter knock-in mouse as screening tool. Transgenic Res 18: 889-897, 2009.

36. Hanahan D and Weinberg RA: Hallmarks of cancer: the next generation. Cell 144: 646-674, 2011.

37. Wicki A, Lehembre F, Wick N, Hantusch B, Kerjaschki D and Christofori G: Tumor invasion in the absence of epithelialmesenchymal transition: podoplanin-mediated remodeling of the actin cytoskeleton. Cancer Cell 9: 261-272, 2006.

38. Chou JL, Chen LY, Lai HC and Chan MW: TGF- $\beta$ : friend or foe? The role of TGF- $\beta /$ SMAD signaling in epigenetic silencing of ovarian cancer and its implication in epigenetic therapy. Expert Opin Ther Targets 14: 1213-1223, 2010. 